\title{
REINFORCEMENT STABILITY AND FRACTURE OF COVER CONCRETE IN RC MEMBERS
}

\author{
By Rajesh Prasad Dhakal ${ }^{1}$ and Koichi Maekawa ${ }^{2}$
}

\begin{abstract}
The main aim of this study is to propose simple and reliable method to predict the buckling length of longitudinal reinforcing bars and also to predict the spalling of cover concrete in reinforced concrete members. Stability analysis is conducted giving due consideration to both geometrical and mechanical properties of the longitudinal reinforcing bars and lateral ties. The tie stiffness required to hold longitudinal reinforcing bars in different buckling modes is derived from energy principles, and it is compared with actual tie stiffness to determine the stable buckling mode. The buckling length is computed as the product of the stable buckling mode and the tie spacing. The proposed buckling length determination method is experimentally verified for various cases. A design method for lateral ties to avoid buckling induced strength degradation is also recommended. The effect of lateral deformation of longitudinal bars is quantitatively evaluated and incorporated in the simulation of cover concrete spalling. Analytical prediction considering spalling and buckling according to the proposed methods showed better agreement with the experimental result.
\end{abstract}

Key words: buckling length, cover concrete, lateral deformation, lateral ties, reinforcing bar, spalling, stable buckling mode, stiffness

\section{INTRODUCTION}

During earthquakes, reinforced concrete members may experience significant lateral deformation of the longitudinal reinforcing bars accompanied by spalling of cover concrete due to large compressive strain. Analytical models neglecting these inelastic material mechanisms cannot capture the post-peak softening behavior accurately, and will consequently overestimate the response ductility (Suda et al. 1996). Hence, average stress-strain relationships of concrete and reinforcing bar including spalling and buckling mechanisms are needed.

It is commonly assumed that the behaviors of reinforcing bar in tension and in compression are similar. In reality, average behavior in compression is different from that in tension. This difference is mainly attributed to the geometrical nonlinearity associated with large lateral deformation of buckled reinforcing bars. Various average compressive stress-strain relationships including buckling (Monti and Nuti 1992; Gomes and Appleton 1997; Rodriguez et al. 1999) have been proposed based on experimental and/or analytical studies of bare bar under axial compression. All of these relationships implicitly or explicitly suggest that the average compressive response of bare bar is a function of length to diameter ratio. For practical application in reinforced concrete members with a system of lateral ties, the bar length used in such bare bar constitutive relations should be replaced with the buckling length of longitudinal reinforcing bar. Hence, the potential buckling length should be pre-determined considering geometrical and mechanical properties of reinforcing bars and cover concrete spalling that may also affect the reinforcement stability.

Longitudinal reinforcing bars inside RC members, when subjected to large compressive strain, undergo lateral deformation. This behavior is referred to as buckling, and is mainly associated with geometrical nonlinearity. Similarly, the authors have defined spalling as the detachment of a part of cover concrete from the core concrete, finally losing its load carrying capacity. Because of their interdependency, these two mechanisms should be considered simultaneously and separating them may lead to incorrect outcome. The formulation of an average compressive stress-strain relationship applicable to longitudinal reinforcing bars in RC members with lateral ties consists of three parts: 1) formulation of a bare-bar average model including buckling; 2) incorporating the effect of lateral ties on the stability of longitudinal reinforcing bar; and 3) accounting for the interaction between cover concrete spalling and reinforcement buckling. An average stress-strain relationship for bare-bar including buckling is formulated and verified by the authors (Dhakal 2000). Hence, this paper focuses mainly on the latter two parts.

\section{DETERMINATION OF BUCKLING LENGTH \\ Effect of Lateral Ties in Buckling Length}

As mentioned earlier, one parameter that governs the average compressive behavior of longitudinal reinforcing bar is the buckling length. The assumption that buckling length of longitudinal reinforcing bars inside an RC member is equal to the spacing of lateral ties does not hold true except in some special cases, such as when: 1) lateral ties are very stiff; 2) longitudinal reinforcing bars are very slender; and 3) tie spacing is very large. Previous studies in this regard (Bresler and Gilbert 1961; Scribner 1986; Papia et al. 1988; Pantazopoulou 1998) were directed towards the

\footnotetext{
${ }^{1}$ Research Fellow, School of Civil and Structural Engineering, Nanyang Technological University, 50 Nanyang Avenue, Singapore 639798, E-mail: cdhakal@ntu.edu.sg (Formerly: Graduate Student, The University of Tokyo)

${ }^{2}$ Professor, School of Civil Engineering, The University of Tokyo, Hongo 7-3-1, Bunkyo-Ku, Tokyo 113, Japan, E-mail: maekawa@concrete.t.u-tokyo.ac.jp
} 
stability-based design of transverse reinforcement in flexural RC members based on some contradictory assumptions regarding the relationship between tie spacing and the buckling length. Bresler and Gilbert (1961) assumed that the lateral ties are sufficiently rigid to prevent lateral displacement of longitudinal bar at the tie locations, and replaced the buckling length with tie spacing in order to determine the size of lateral ties. In contrast, Scribner (1986) reported that the plastic hinge in members subjected to repeated reverse inelastic flexure spans through a length equal to overall beam depth. By assuming that the tie spacing is equal to one-fourth of beam depth, it was concluded that the buckling length extends through three tie spacings.

However, axial compression tests of reinforced concrete prisms (Kato et al. 1995) showed that the buckling length varies from one to several times tie spacing depending on the geometrical and mechanical properties of lateral ties and longitudinal bar. If the size and spacing of lateral ties are properly designed to provide a rigid support to the longitudinal reinforcing bar at the tie locations, buckling confines between two adjacent stirrups. It is to be noted that if the buckling length changes from one to two times tie spacing, the length to diameter ratio will be doubled, and the average compressive stress-strain relationship of reinforcement over the buckling length domain is significantly changed (Dhakal 2000). As the average compressive response of reinforcing bar is very sensitive to the buckling length, simplified assumptions regarding buckling length might lead to significantly inaccurate reinforcement behavior. Here, a theoretical method to determine the buckling length of longitudinal reinforcing bars inside an RC member, which is equal to the integral multiple of tie spacing, is proposed based on stability analysis.

\section{Assumptions and General Flow of Computation}

The entire process of buckling length determination is illustrated with a flow-chart in Fig. 1. First, the actual stiffness of a lateral tie effective to each longitudinal reinforcing bar connected to the tie is computed. Next, the minimum transverse stiffness at the tie locations required to hold a longitudinal reinforcing bar in different buckling modes is determined using energy principles. Here, buckling mode refers to the number of tie spacing covered by the buckling length. If the effective tie stiffness is less than the required stiffness for mode $n-1$ but exceeds that for mode $n$, the provided system of lateral ties can hold the longitudinal reinforcing bars in the $n^{\text {th }}$ buckling mode. In other words, $n$ is the stable buckling mode and multiplying it by the tie spacing gives the buckling length for the given combination of longitudinal and transverse reinforcement. In derivations based on force equilibrium, the complex kinematics of the system needs to be solved to compute the forces on lateral ties. However, utilizing the stiffnessbased approach described in this paper enables the complexities involved with the numerical implementation of the equilibrium equations to be removed, and does not require the simplifications commonly used in similar derivations.

Longitudinal reinforcing bar is simulated as flexural member fixed at both ends of buckling length to represent the restraining mechanism of lateral ties at these locations. As shown in Fig. 2, a cosine curve satisfying the fixed boundary condition is adopted to define the deformational shape of buckled bar. As the lateral deformation of the buckled bar increases, some sections within the buckling length undergo compression hardening and some experience unloading (Dhakal 2000). As the unloading stiffness is equal to the elastic modulus $E_{s}$, the flexural rigidity of unloading sections is equal to the elastic rigidity $E_{s} I$, where $I$ is the moment of inertia of the bar crosssection. On the other hand, flexural rigidity of hardening sections is significantly smaller than the elastic rigidity due primarily to much reduced stiffness in hardening. Hence, the average flexural rigidity within the buckling length is undoubtedly less than the elastic rigidity, but its accurate estimation is difficult due to complex mechanisms involved. The average flexural rigidity of the longitudinal bar is also influenced by its yield strength. As the associated plasticity is less and the secant stiffness is higher in high strength bars, the average flexural rigidity increases with increase in yield strength and vice versa. Finally, considering the qualitative influence of nonlinear strain distribution and yield strength, the average flexural rigidity of main bar is expressed as $\mathrm{EI}=0.5 \mathrm{E}_{\mathrm{s}} \mathrm{I} \sqrt{ }\left(\mathrm{f}_{\mathrm{y}} / 400\right)$, where $f_{y}$ is the yield strength expressed in Mpa. In fact, this approximation closely represents the actual behavior, as will be justified later through experimental verification.

The lateral ties are simulated by discrete elastic springs. In reality, lateral ties show elasto-plastic behavior and its tangential stiffness reduces nearly to zero after yielding. Ties around the middle of the buckling length are more likely to yield due to larger lateral deformation of the longitudinal bars. Strictly speaking, lateral deformation at each tie position should be exactly computed and if it exceeds the yielding deformation, corresponding spring should be eliminated from the system. However, to obtain a reliable approximation, a parametric study is conducted in this study with the springs in central L/2, L/3, and L/4 regions eliminated from the system. The expansion of core concrete in compression, which induced tensile strain in lateral ties, is not explicitly considered in the formulation. The lateral force in the tie, which counterbalances the outward thrust from the confined core concrete, is accounted for by including the spring energy in the computation. Experimental and analytical investigations (Irawan and Maekawa 1994) have proved that the tensile strain induced in the lateral ties due to core-concrete expansion is less 
than yielding strain except for axially compressed RC member with spiral hoops. Consequently, the stiffness of the lateral ties will remain elastic in spite of the core concrete expansion, thus justifying the simulation.

\section{Formulation}

As shown in Fig. 2, the derivation of the required spring stiffness $k_{n}$ corresponding to an arbitrary mode $n$ should address two consecutive deformational modes $n$ and $n+1$, as it tries to avoid the $(n+1)^{\text {th }}$ mode and to sustain the $n^{\text {th }}$ mode. The lower modes need not be considered because they are already checked in the previous steps and proved not to exist. First, a term $U$, which is expressed as the sum of the energies $U_{n}$ and $U_{n+1}$ associated with the two buckling modes $n$ and $n+1$, is introduced in (1). As shown in (2) and (3), energy corresponding to each buckling mode includes the strain energy, energy stored in the springs and the energy due to shortening of reinforcing bar.

$$
\begin{aligned}
& U=U_{n}+U_{n+1} \\
& U_{n}=\int_{0}^{n s} \frac{E I}{2}\left(\frac{d^{2} y_{n}}{d x^{2}}\right)^{2} d x+c_{i} \sum_{i=1}^{n} \frac{k_{n}}{2} y_{n}^{i s^{2}}-\int_{0}^{n s} \frac{P_{n}}{2}\left(\frac{d y_{n}}{d x}\right)^{2} d x \\
& U_{n+1}=\int_{0}^{(n+1) s} \frac{E I}{2}\left(\frac{d^{2} y_{n+1}}{d x^{2}}\right)^{2} d x+c_{i} \sum_{i=1}^{n+1} \frac{k_{n}}{2} y_{n+1}^{i s}-\int_{0}^{2} \frac{P_{n}}{2}\left(\frac{d y_{n+1}}{d x}\right)^{2} d x
\end{aligned}
$$

Here, $c_{i}$ is a coefficient to incorporate the plasticity of lateral ties and its value is 0 for the eliminated springs and 1 for the rest. Similarly, $k_{n}$ and $P_{n}$ are the critical spring stiffness and the axial load corresponding to the $\mathrm{n}^{\text {th }}$ mode, respectively. Now, using the prescribed deformational shapes and minimizing $U$ with respect to each of the maximum amplitudes $a_{n}$ and $a_{n+1}$, (4) and (5) can be obtained.

$$
\begin{aligned}
& \frac{\partial U}{\partial a_{n}}=0 \Rightarrow \frac{2 \pi^{4} E I}{n^{3} s^{3}}+\frac{c_{i} k_{n}}{4} \sum_{i=1}^{n}\left(1-\cos \frac{2 i \pi}{n}\right)^{2}-\frac{P_{n} \pi^{2}}{2 n s}=0 \\
& \frac{\partial U}{\partial a_{n+1}}=0 \Rightarrow \frac{2 \pi^{4} E I}{(n+1)^{3} s^{3}}+\frac{c_{i} k_{n}}{4} \sum_{i=1}^{n+1}\left(1-\cos \frac{2 i \pi}{n+1}\right)^{2}-\frac{P_{n} \pi^{2}}{2(n+1) s}=0
\end{aligned}
$$

These two simultaneous equations finally yield the required spring stiffness $k_{n}$ and the corresponding load $P_{n}$. The required spring stiffness, computed for different buckling modes corresponding to the three different sets of eliminated springs, is shown in Table 1 . The equivalent stiffness $k_{\text {eq }}$, mentioned in the table is a dimensionless parameter and multiplying it by $\pi^{4} \mathrm{EI} / \mathrm{s}^{3}$ gives the spring stiffness $k_{n}$ required to stabilize the longitudinal bar in the corresponding buckling mode. As expected, the required stiffness becomes smaller for higher buckling modes. It can be noticed that the range of eliminated springs does not influence the result except for some special buckling modes, which is contributed by the ambiguity arisen due primarily to the existence of spring exactly at the boundary of the assumed yield zone. Hence, average of the equivalent stiffness corresponding to the former two cases (springs within central $\mathrm{L} / 2$ and $\mathrm{L} / 3$ eliminated) is recommended for further use (Table 1 ).

\section{Stiffness of Lateral Ties}

The buckling tendency of longitudinal bars induces axial tension in the tie-legs along the buckling direction. Hence, the resistance provided by the lateral ties against buckling of longitudinal bar is mainly contributed by the axial stiffness of these tie-legs. The axial stiffness of each tie leg is $\mathrm{E}_{\mathrm{t}} \mathrm{A}_{\mathrm{t}} / \mathrm{l}_{\mathrm{e}}$, where $E_{t}, A_{t}$ and $l_{e}$ are elastic modulus, cross-sectional area and the leg-length, respectively. Assuming that the total stiffness of $n_{l}$ tie-legs along the buckling direction contribute equally to $n_{b}$ longitudinal bars that are prone to simultaneous buckling, the restraining stiffness of the tie system effective against buckling of each longitudinal bar can be calculated using (6).

$k_{t}=\frac{E_{t} A_{t}}{l_{e}} \times \frac{n_{l}}{n_{b}}$ 
The values of $n_{l}$ and $n_{b}$ for some common arrangements of longitudinal and lateral reinforcement are illustrated in Fig. 3. The values shown in Fig. 3 are for flexural loading, where only the reinforcing bars in compressive side are prone to simultaneous buckling. Nevertheless, in case of concentric axial compression, all bars have equal strain and bars in both sides of the tie legs tend to buckle at the same time. Hence, $n_{b}$ and $n_{l}$ along each axis should be determined considering the tie-legs parallel to the axis and longitudinal bars in both sides of the tie-legs.

\section{Comparison with Experimental Results}

For experimental verification, an axially loaded prism and a laterally loaded flexural column are considered. The specimen details are illustrated in Fig. 4, and the computations of stable buckling modes according to the proposed method are presented in Table 2. Computations in Table 2 yield stable buckling modes of 1 for the prism and 3 for the flexural column, which resemble with the experimental observation shown in Fig. 4.

For further verification, the predictions according to the proposed method are compared with 45 experimental observations (Bresler and Gilbert 1961; Scribner 1986; Kato et al. 1995; PWRI 1999) including some experiments by the authors. These cases include compression tests of prisms as well as bending tests of beams and columns reinforced with normal strength and high strength steel bars. The experimental parameters and the computation of buckling mode are presented in Table 3. Comparative curves relating the equivalent stiffness and the predicted as well as experimentally observed buckling modes are shown in Fig. 5. Furthermore, the accuracy of the proposed method, in terms of the difference between observed and predicted buckling modes, is also depicted in Fig. 5. As can be seen in the illustrations, the proposed method is in very good agreement with the experimental observations, and even the higher buckling modes could be satisfactorily predicted. Hence, the approximation used in computing average flexural rigidity of reinforcing bars is justified.

\section{DESIGN RECOMMENDATION FOR LATERAL TIES AGAINST BUCKLING}

Lateral ties are commonly designed to provide additional shear resistance and confinement to the core concrete. However, the contribution of lateral ties in resisting buckling of longitudinal bars is seldom considered. The authors believe that properly designed lateral ties can successfully restrict buckling of longitudinal bars, and consequently improve the post peak response and ductility. If the value of a parameter $L / D \sqrt{ }\left(f_{y} / 100\right)$ is less than or equal to 8 , premature buckling can be avoided and the average compressive response of longitudinal bar is stable (Dhakal 2000). Accordingly, lateral ties can be designed using the following steps to avoid buckling-induced strength degradation in the post-peak region.

1. Design lateral ties (spacing $s$ and the diameter $D$ ) according to the existing shear strength criteria.

2. Compute $s / D \sqrt{ }\left(f_{y} / 100\right)$. If it is more than 8 , reduce the spacing so that $s / D \sqrt{ }\left(f_{y} / 100\right)$ is less than or equal to 8 .

3. If it is less than 8 , find the largest possible buckling mode $n$ so that the value of ns/D $\sqrt{ }\left(\mathrm{f}_{\mathrm{y}} / 100\right)$ is not more than 8 .

4. Compute the axial tie stiffness $k_{t}$ effective to each longitudinal bar according to (6).

5. Compare the ratio $\mathrm{k}_{\mathrm{t}} /\left(\pi^{4} \mathrm{EIs}^{3}\right)$ with the equivalent required stiffness $k_{e q}$ corresponding to mode $n$ determined in step 3 from Table 1.

6. If the ratio is greater than $k_{e q}$ corresponding to mode $n$, current system of lateral ties is capable of avoiding premature buckling of longitudinal reinforcing bars.

7. If the ratio is less than $k_{\text {eq }}$, either the diameter should be increased or the spacing should be reduced. Step 2 to step 6 should be repeated until the ratio $\mathrm{k}_{\mathrm{t}} /\left(\pi^{4} \mathrm{EIs}^{3}\right)$ becomes greater than $k_{\text {eq }}$ corresponding to mode $n$.

\section{COVER CONCRETE SPALLING}

\section{Tension Softening due to Splitting Crack}

When a flexural RC member is subjected to high moment, the cover concrete in the compression side suddenly spalls off and looses further load-carrying capacity. Hence for analytical modeling, it is important to know when the cover concrete spalls so that its contribution in the overall response is accounted for. In this study, an analytical method to determine the compressive strain of nearby longitudinal reinforcing bar corresponding to cover concrete spalling is proposed.

The tensile capacity of cover concrete in transverse direction decreases with increase in the splitting crack width, which can be described by bilinear tension softening model (Fig. 6). The area enclosed by the curve gives fracture energy, which is defined as the energy required to create a perfect crack of unit area that cannot transfer the normal stress anymore. As the fracture energy is considered to be a material constant depending on many conditions, it should be determined by experiments. The splitting crack width corresponding to zero tensile capacity can be calculated using the fracture energy $G_{F}$ and the tensile strength $f_{t}$ of cover concrete (Fig. 6). A splitting crack of this width should be formed to cause complete spalling of the cover concrete. 


\section{Factors Influencing Cover Concrete Spalling}

Two factors contribute to the formation of the splitting crack leading to cover concrete spalling. First, the compressive strain deteriorates the cover concrete creating some vertical cracks, due to which the tensile capacity in transverse direction is reduced. Next, the buckling tendency of longitudinal bar widens these cracks, finally reducing the tensile capacity to zero and separating the cover concrete from the core concrete. Here, the equivalent damage due to axial compressive strain is represented by fracture parameter $K$, which was originally proposed (Maekawa and Okamura 1983) to account for the stiffness degradation of concrete due to fracture. For normal concrete, the fracture parameter is calculated as in (7), where $E$ is the ratio of compressive strain to the peak strain of concrete.

$$
K=\exp (-0.73 E(1-\exp (-1.25 E))) ; \quad 1.0 \geq K \geq 0.25
$$

As shown in Fig. 6, the splitting crack width required to cause cover concrete spalling consists of two parts: 1) $(1-\mathrm{K}) \mathrm{G}_{\mathrm{F}} / \mathrm{f}_{\mathrm{t}}$, representing the equivalent splitting crack width due to axial compressive strain; and 2$)(4+\mathrm{K}) \mathrm{G}_{\mathrm{F}} / \mathrm{f}_{\mathrm{t}}$, representing the equivalent crack widening due to reinforcement buckling. Assuming the maximum lateral deformation of the longitudinal reinforcing bar at the center of the buckling length $a$ to be equal to the widening of splitting crack, the critical lateral deformation $a_{c r}$ required to cause complete spalling of cover concrete can be expressed as in (8).

$$
a_{c r}=(4+K) \frac{G_{F}}{f_{t}}
$$

\section{Determination of Spalling Strain}

Longitudinal reinforcing bars undergo axial shortening under elastic compression, and the plastic compressive strain is accompanied by lateral deformation. Fig. 7 illustrates the relationship between the plastic compressive strain $\varepsilon_{p}$ and the maximum lateral deformation $a$. In fact, elastic shortening is excluded from the buckling length $L$, and the plastic strain will be later added to the elastic part to obtain total strain. In the figure, $\lambda$ is the projected length of laterally deformed bar and can be computed as $\mathrm{L}\left(1-\varepsilon_{\mathrm{p}}\right)$. Hence, the integration of the deformed length over the range $\lambda$ gives the original length $L$, as in (9).

$L=\int_{0}^{\lambda} d s=\int_{0}^{\lambda} \sqrt{1+\left(\frac{d y}{d x}\right)^{2}} d x=2 \int_{0}^{\lambda / 2} \sqrt{1+\left(\frac{a \pi}{\lambda} \sin \frac{2 \pi x}{\lambda}\right)^{2}} d x$

Using Taylor's expansion for the square root term, and neglecting higher order terms of $a^{2} / \lambda^{2}$, we obtain (10).

$L=2 \int_{0}^{\lambda / 2}\left(1+\frac{a^{2} \pi^{2}}{2 \lambda^{2}} \sin ^{2} \frac{2 \pi x}{\lambda}\right) d x=\frac{4 \lambda^{2}+a^{2} \pi^{2}}{4 \lambda}$

Replacing $\lambda$ with $\mathrm{L}\left(1-\varepsilon_{\mathrm{p}}\right)$, and neglecting higher order terms of $\varepsilon_{p}$ gives (11).

$\varepsilon_{p}=\frac{a^{2} \pi^{2}}{4 L^{2}}$

When $a$ in (11) is replaced with its critical value $a_{c r}$, we get (12).

$\varepsilon_{p}^{s p}=\frac{a_{c r}^{2} \pi^{2}}{4 L^{2}}$ 
Here, $\varepsilon_{p}^{s p}$ is the plastic compressive strain of longitudinal reinforcing bar required to cause cover spalling, and adding it with the yield strain gives the total compressive strain corresponding to the complete spalling of cover concrete. Strictly speaking, (12) holds only for the monotonic case (absolute compressive strain), and the loading history must be considered for cases in which tensile loading may have been applied.

\section{APPLICATION AND VERIFICATION \\ Experimental Setup and Specimen Details}

A reinforced concrete cantilever column was tested to investigate the reliability of the proposed models in predicting the post-peak response. The experimental setup and the specimen layout are shown in Fig. 8, and the geometrical and mechanical properties of the specimen are listed in Table 4. The column is intentionally provided with significant axial compression (14\% of the axial capacity) and thick cover concrete $(30 \mathrm{~mm})$ to highlight the influence of spalling and buckling. In order to avoid shear failure, the column was designed so that the shear capacity is higher than the bending capacity. The rigid footing, which was cast monolithically with the column, was tightly fixed to the base slab with prestressing tendons to ensure the cantilever mechanism. Two actuators of a triaxial loading machine were used to apply simultaneously cyclic lateral displacement at $120 \mathrm{~cm}$ from the footing top and an axial compression at the top of the column.

\section{Material Models and Analytical Simulation}

To verify the applicability of proposed methods in FEM analysis, the computed buckling length is used in a bare-bar buckling model (Dhakal 2000), and the spalling criterion is supplemented with the elasto-plastic and fracture model (Maekawa and Okamura 1983). These enhanced models are installed in a finite element analysis program COM3 [Concrete Model in 3D] (Hauke and Maekawa 1999), which is used for analyzing the tested RC column. The RC column is represented by frame elements, and is analyzed by fiber technique (Menegotto and Pinto 1973). In fiber technique, the member cross-section is divided into many cells, and each element consisting of several longitudinal fibers is represented by its centerline. The strain of each fiber is calculated based on the EulerKirchoff's hypothesis; i.e. plane section remains plane after bending. The response of each element is the integration of all fiber responses that are computed based on average constitutive models of the materials in the corresponding fibers. Fiber technique and material models used for concrete and reinforcing bar are schematically illustrated in Fig. 9. These material models are fully path dependent and take into account the steel-concrete bond and loading rate effect in the concrete response. The details of these models and their verification for RC members subjected to static and dynamic loading are elaborated in a book by Okamura and Maekawa (1991).

The rigid footing is represented by a fixed support at the base of the column. The RC column is discretized into five elements and the cross-section is divided into 221 cells. To simulate cover spalling, the stress transferred by concrete fibers outside the lateral tie is reduced to zero once the spalling strain is reached in nearby reinforcing bars. To incorporate P-delta effect, geometrical nonlinearity is given due consideration in the analysis. Due to substantial axial load, the pullout of reinforcing bars from column-footing joint was negligible in the experiment, and is hence neglected in the analysis.

\section{Results and Discussions}

As the column was designed to have comparatively higher shear strength, no diagonal shear crack could be seen. In experiment, uniform flexural cracks appeared gradually, and cover spalling at the column base could be noticed after a few loading cycles when the applied displacement reached around $15 \mathrm{~mm}$. However, the buckling displacement could not be distinguished in experiment although buckled bars were seen after scratching out the spalled cover concrete. The experimental and analytical load-displacement curves with and without using buckling and spalling models are shown in Fig. 10. As indicated in the figure, the spalling displacement predicted in analysis is also close to $15 \mathrm{~mm}$. Gradual decrease of lateral load can be observed after initiation of cover spalling in the experimental result. In contrast, a sudden drop in the load is seen in the analytical result. This is because the stress carried by cover concrete fibers is abruptly neglected once the spalling criterion is fulfilled. Hence, this abrupt reduction in the lateral load is the overall contribution of spalling, and is carried over throughout the post-spalling phase. In the analysis, buckling took place during the last loading cycle, after which additional reduction in the lateral load could be observed. The difference between the analytically predicted lateral loads minus the spalling induced sudden drop is the contribution of buckling, which increases gradually with the applied displacement.

Although the load-displacement curve predicted without considering spalling and buckling exhibits mild postpeak softening that is due primarily to the P-delta effect, lateral load in the post-peak region is much higher than in experimental result. Incorporating buckling and spalling models in the analysis significantly improved the agreement between the analytical and experimental results, and the enhanced FEM analysis could reliably predict the post-peak 
softening behavior as well. The ultimate deformation, defined as the displacement in post-peak region corresponding to either yield load or $80 \%$ of the peak load depending on design codes followed, is significantly overestimated if these inelastic material mechanisms are overlooked. As yielding displacement is not much influenced by spalling and buckling, the response ductility, which is an important parameter in seismic design, is also overestimated. This research is hence useful to practitioners engaged in seismic design, as it enlightens the mechanisms that may impair the seismic performance of RC structures.

\section{CONCLUSIONS}

An analytical method to determine buckling length of longitudinal reinforcing bars inside reinforced concrete members was proposed. Comparison with several experimental observations revealed ample evidence of the reliability of this method. Moreover, enhanced design method of lateral ties to avoid premature buckling of longitudinal bar was also proposed. The deterioration of cover concrete due to axial compressive strain and widening of splitting cracks due to buckling tendency of longitudinal bar were separately considered in the derivation of spalling criterion. The proposed buckling length computation and spalling criterion were used to carry out finite element analysis of a laterally loaded cantilever RC column under axial compression. The analytical results were in good agreement with the experimental results, which further verified the validity of the proposed models.

\section{ACKNOWLEDGEMENTS}

The authors gratefully acknowledge TEPCO Research Foundation and Grant-in-aid for scientific research No. 11355021 for providing financial support to accomplish this research.

\section{APPENDIX. REFERENCES}

Bresler, B., and Gilbert, P. H. (1961). "Tie requirements for reinforced concrete columns. " Journal of ACI, 58 (5), 555-570.

Dhakal, R. P. (2000). " Enhanced fiber model in highly inelastic range and seismic performance assessment of reinforced concrete." Doctoral Dissertation, Department of Civil Engineering, The University of Tokyo.

Gomes, A., and Appleton, J. (1997). "Nonlinear cyclic stress-strain relationship of reinforcing bar including buckling." Engineering Structures, 19 (10), 822-826.

Hauke, B., and Maekawa, K. (1999). "Three-dimensional modeling of reinforced concrete with multi-directional cracking." Journal of Materials, Concrete Structures and Pavements, JSCE, 634 (45), 349-368.

Irawan, P., and Maekawa, K. (1994). "Three-dimensional analysis of strength and deformation of confined concrete columns." Concrete Library, JSCE, 24, 47-70.

Kato, D., Kanaya, J., and Wakatsuki, K. (1995). "Buckling strains of main bars in reinforced concrete members." Proc., $5^{\text {th }}$ East Asia and Pacific Conference in Strucural Engineering and Construction EASEC-5, Gold Coast, Australia, 699-704.

Maekawa, K., and Okamura, H. (1983). "The deformational behavior and constitutive equation of concrete using the elasto-plastic and fracture model." Journal of Faculty of Engineering, The University of Tokyo, 37 (2), 253-328.

Menegotto, M., and Pinto, P. E. (1973). "Method of analysis of cyclically loaded RC plane frames including changes in geometry and non-elastic behavior of elements under normal force and bending." Preliminary Report No. 13, International Association of Bridge and Structural Engineering IABSE, Zurich, Switzerland, 15-22.

Monti, G., and Nuti, C. (1992). "Nonlinear cyclic behavior of reinforcing bars including buckling." Journal of Structural Engineering, ASCE, 118 (12), 3268-3284.

Okamura, H., and Maekawa, K. (1991). "Nonlinear analysis and constitutive models of reinforced concrete." The University of Tokyo, ISBN4-7655-1506-0 C 3051, Gihodo Publication, Tokyo, Japan.

Pantazopoulou, S. J. (1998). "Detailing for reinforcement stability in RC members.” Journal of Structural Engineering, ASCE, 124 (6), 623-632.

Papia, M., Russo, G., and Zingone, G. (1988). "Instability of longitudinal bars in RC columns." Journal of Structural Engineering, ASCE, 114 (2), 445-461.

PWRI (1999). "Size effect in the seismic performance of reinforced concrete piers." Technical Memorandum No. 234, Public Work Research Institute PWRI, Tsukuba, Japan (In Japanese).

Rodriguez, M. E., Botero, J. C., and Villa, J. (1999). "Cyclic stress-strain behavior of reinforcing steel including effect of buckling." Journal of Structural Engineering, ASCE, 125 (6), 605-612.

Scribner, C. F. (1986). "Reinforcement buckling in reinforced concrete flexural members." ACI Journal, 83(6), 966-973.

Suda, K., Murayama, Y., Ichinomiya, T., and Shimbo, H. (1996). "Buckling behavior of longitudinal reinforcing bars in concrete column subjected to reverse lateral loading." Proc., $11^{\text {th }}$ World Conference on Earthquake Engineering, Acapulco, Mexico, CD ROM Paper No. 1753.

\section{APPENDIX. NOTATION}

$A_{t}=$ cross-sectional area of lateral tie;

$a=$ maximum amplitude at the center of buckling length;

$a_{c r}=$ critical value of $a$ corresponding to cover spalling;

$c_{i}=$ coefficient to include plasticity of lateral tie; 
$D=$ diameter of longitudinal reinforcing bar;

$E=$ ratio of compressive strain to peak strain of concrete;

$E_{s}=$ Young's modulus of longitudinal reinforcing bar;

$E_{t}=$ Young's modulus of lateral tie;

$E I=$ average flexural rigidity of longitudinal reinforcing bar;

$f_{t}=$ tensile strength of concrete;

$f_{y}=$ yield strength of longitudinal reinforcing bar;

$G_{F}=$ fracture energy of normal concrete;

$I=$ moment of inertia of longitudinal reinforcing bar;

$K=$ fracture parameter in elasto-plastic and fracture model;

$k_{t}=$ axial stiffness of lateral tie;

$k_{e q}=$ equivalent required spring stiffness, $k_{e q}=\mathrm{k}_{\mathrm{n}} \times \mathrm{s}^{3} / \pi^{4} \mathrm{EI}$;

$k_{n}=$ required spring stiffness for the $n^{\text {th }}$ buckling mode;

$L=$ buckling length of longitudinal reinforcing bar;

$l_{e}=$ effective length of tie leg;

$n_{b}=$ number of main bars prone to simultaneous buckling;

$n_{l}=$ number of tie legs along the buckling direction;

$P_{n}=$ axial compressive load corresponding to the $\mathrm{n}^{\text {th }}$ mode;

$s=$ spacing of lateral ties;

$U=$ total potential energy of the system;

$\varepsilon_{p}=$ plastic compressive strain of the reinforcing bar;

$\varepsilon_{p}^{s p}=$ compressive plastic strain required for cover spalling;

$\lambda=$ projected length of buckled reinforcing bar; 


\section{LIST OF FIGURES:}

FIG. 1. Flow-chart of buckling length determination

FIG. 2. Simulation and determination of required spring stiffness

FIG. 3. Values of $n_{b}$ and $n_{l}$ for common reinforcement arrangements

FIG. 4. Buckling modes observed in experiment

FIG. 5. Comparison of proposed method with experimental observations

FIG. 6. Equivalent deterioration of cover concrete due to axial compression

FIG. 7. Effect of reinforcement lateral deformation in cover spalling

FIG. 8. Experimental setup and the geometrical property of the specimen (Unit: $\mathbf{m m}$ )

FIG. 9. Fiber technique and material models used in FEM analysis

FIG. 10. Experimental and analytical results (load-displacement curve) 


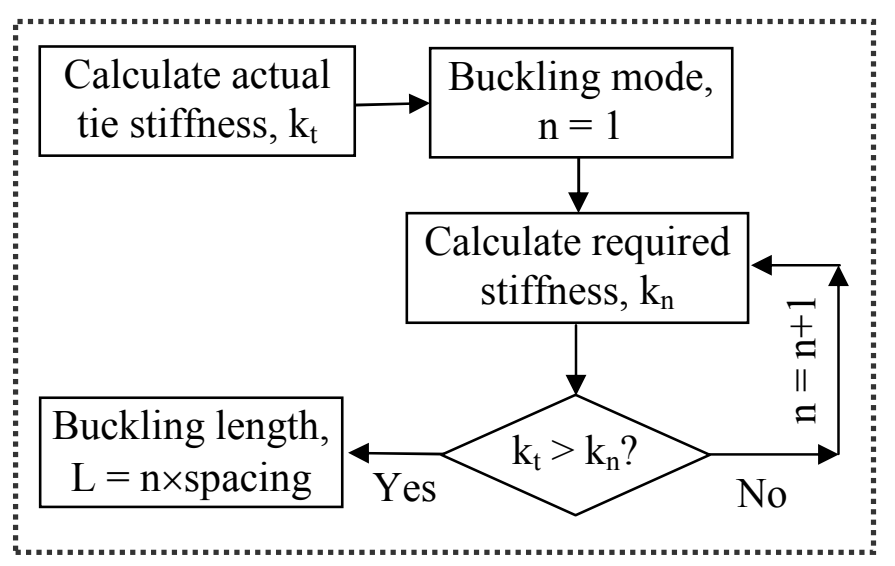




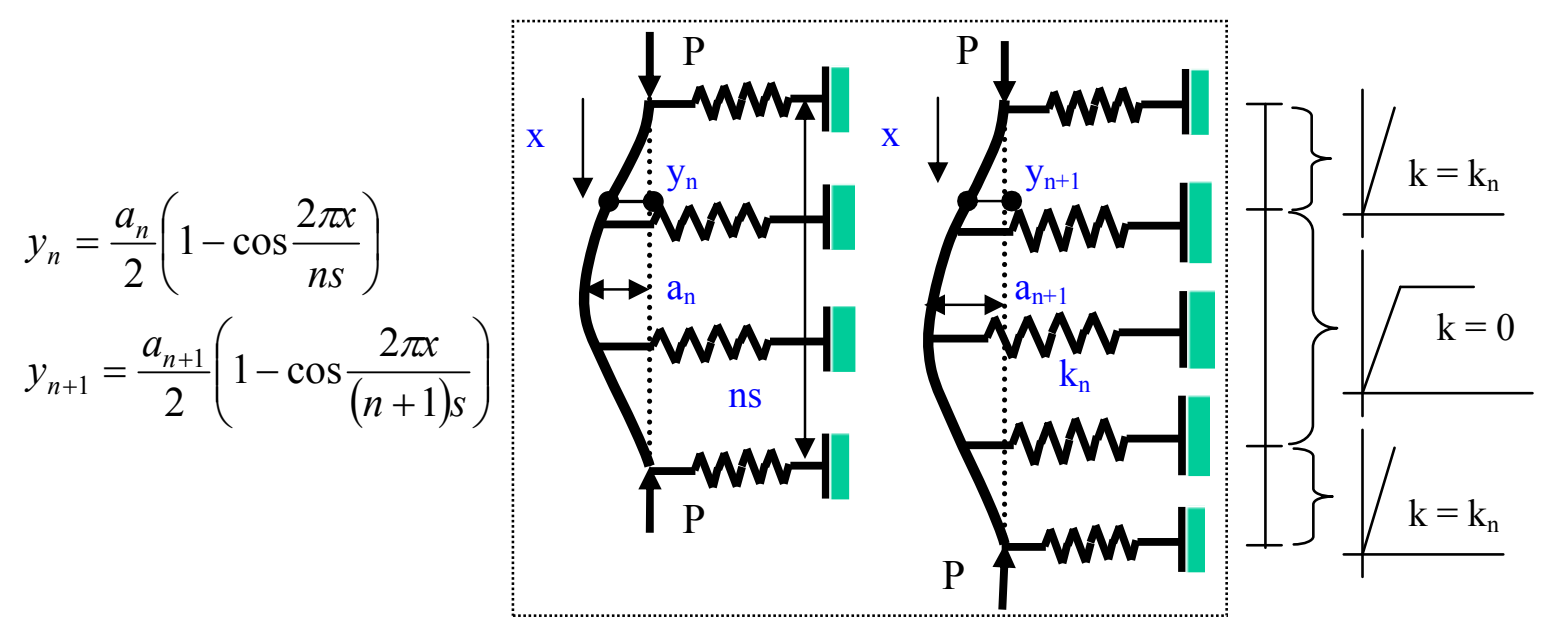




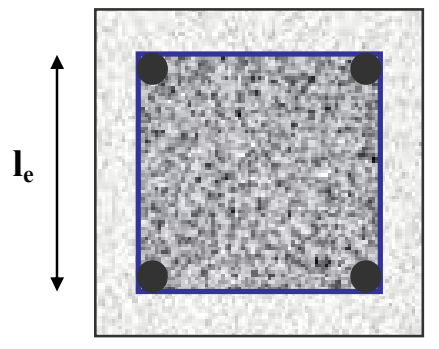

$n_{b}=2 ; n_{l}=2$

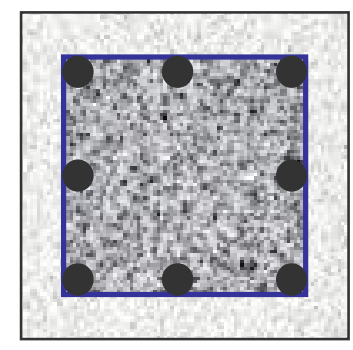

$\mathbf{n}_{\mathrm{b}}=\mathbf{3} ; \mathbf{n}_{\mathbf{l}}=\mathbf{2}$

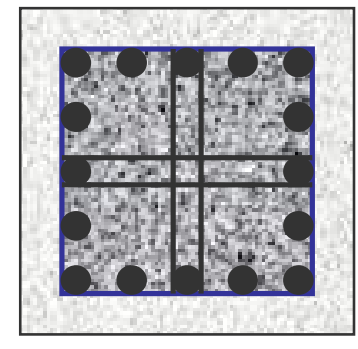

$\mathrm{n}_{\mathrm{b}}=5 ; \mathrm{n}_{\mathrm{l}}=4$

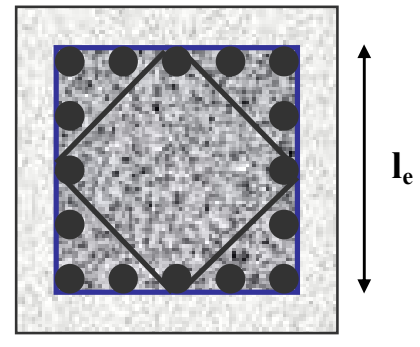

$n_{b}=5 ; n_{1}=4$

$I_{e}$ 


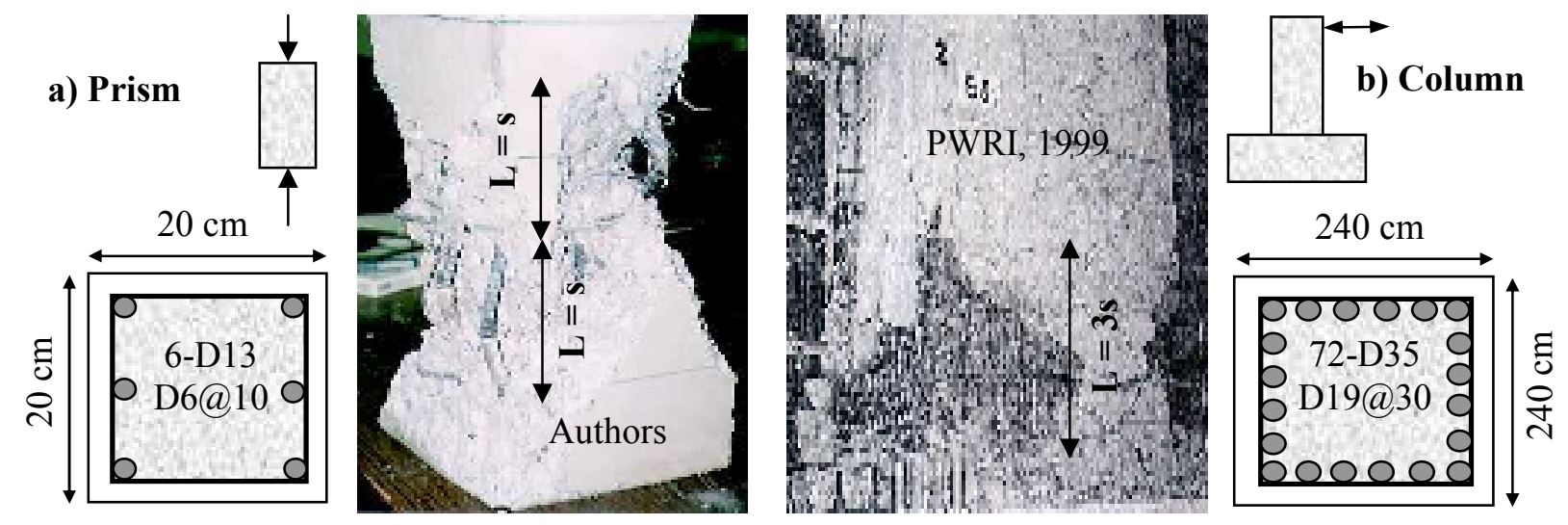



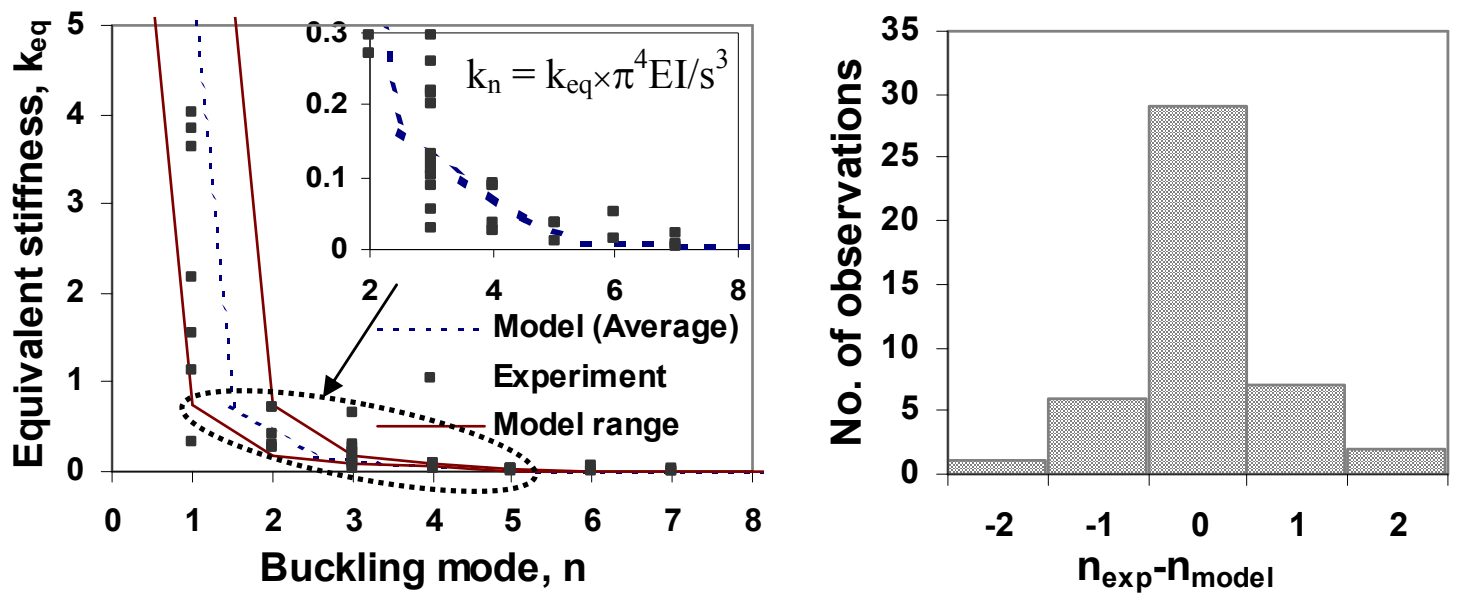


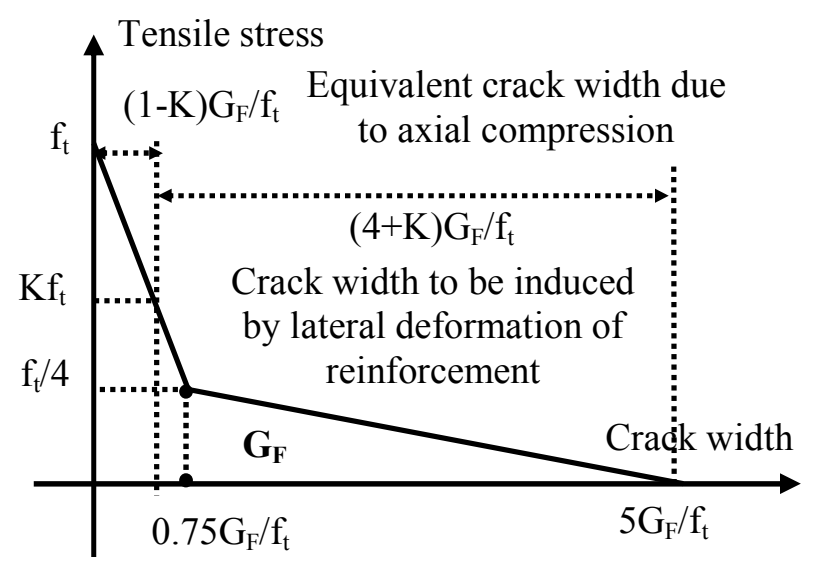




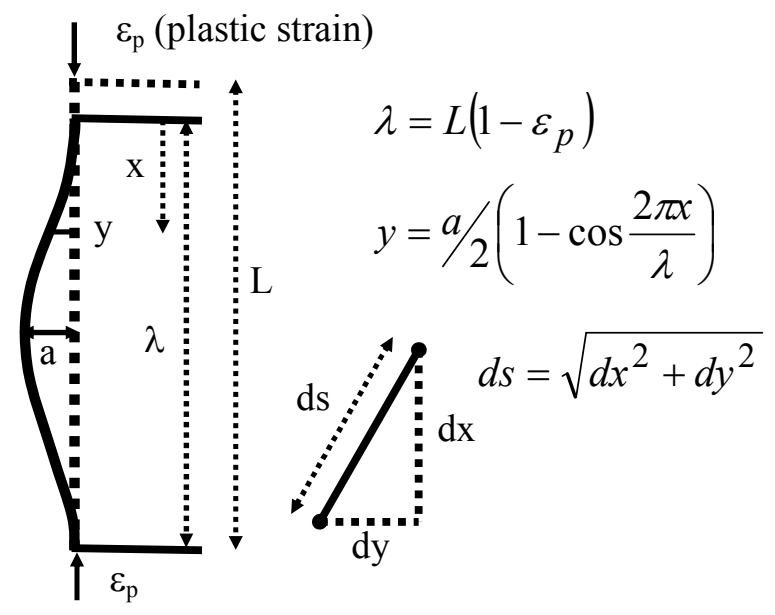



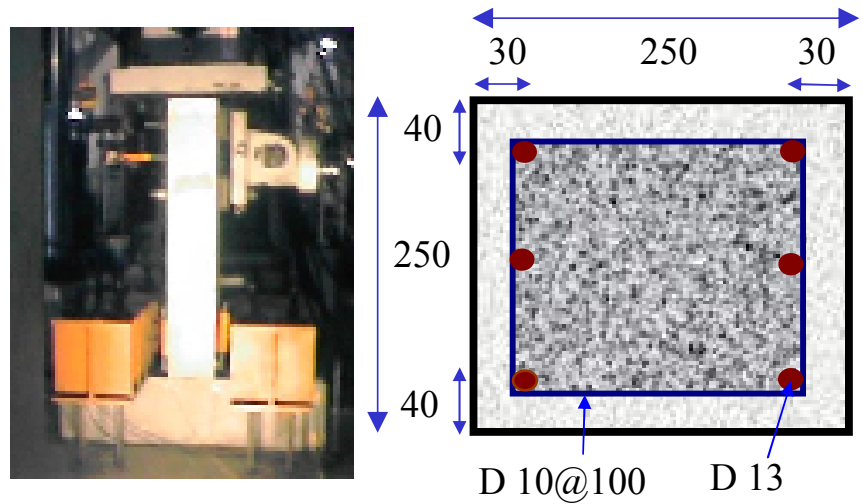


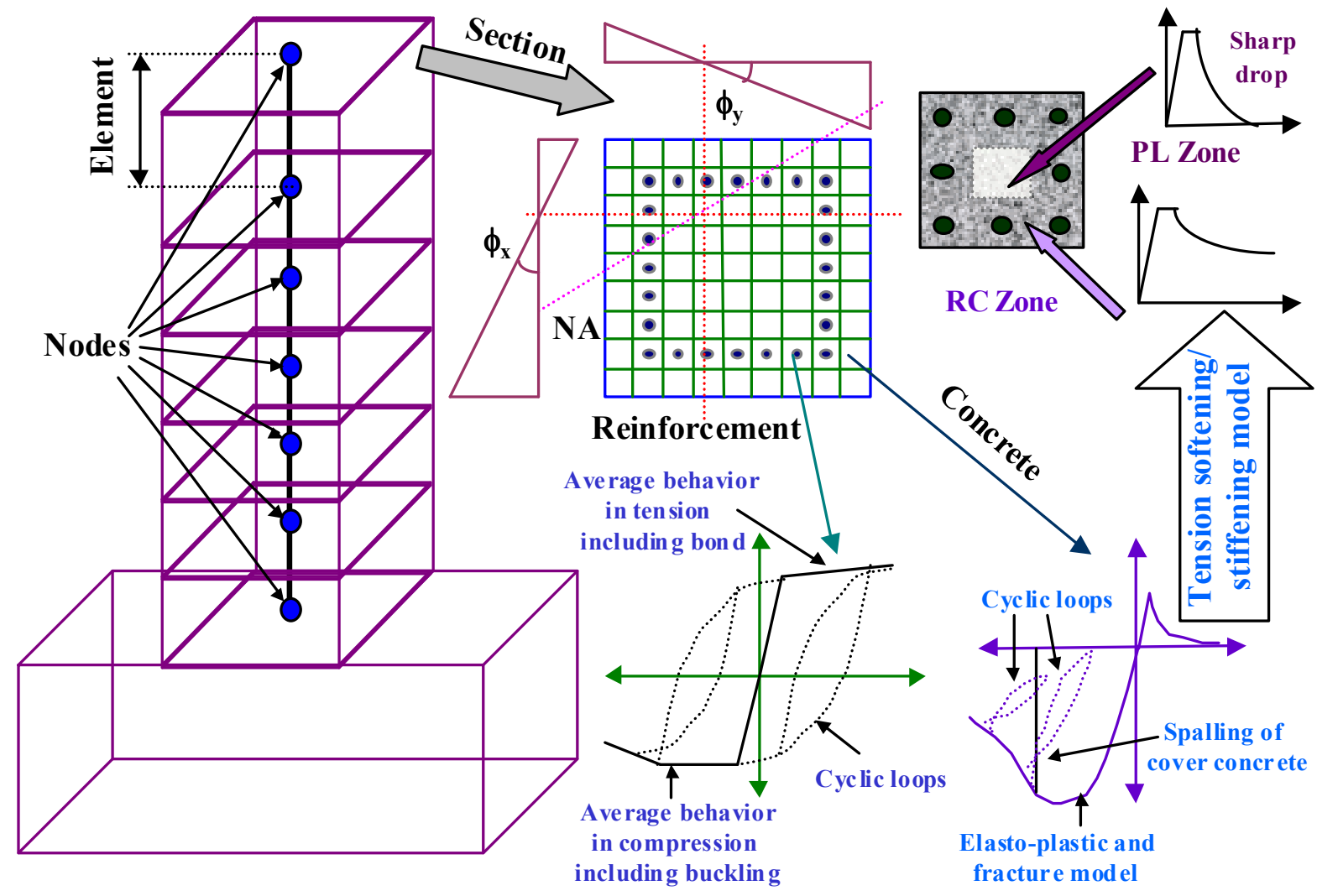



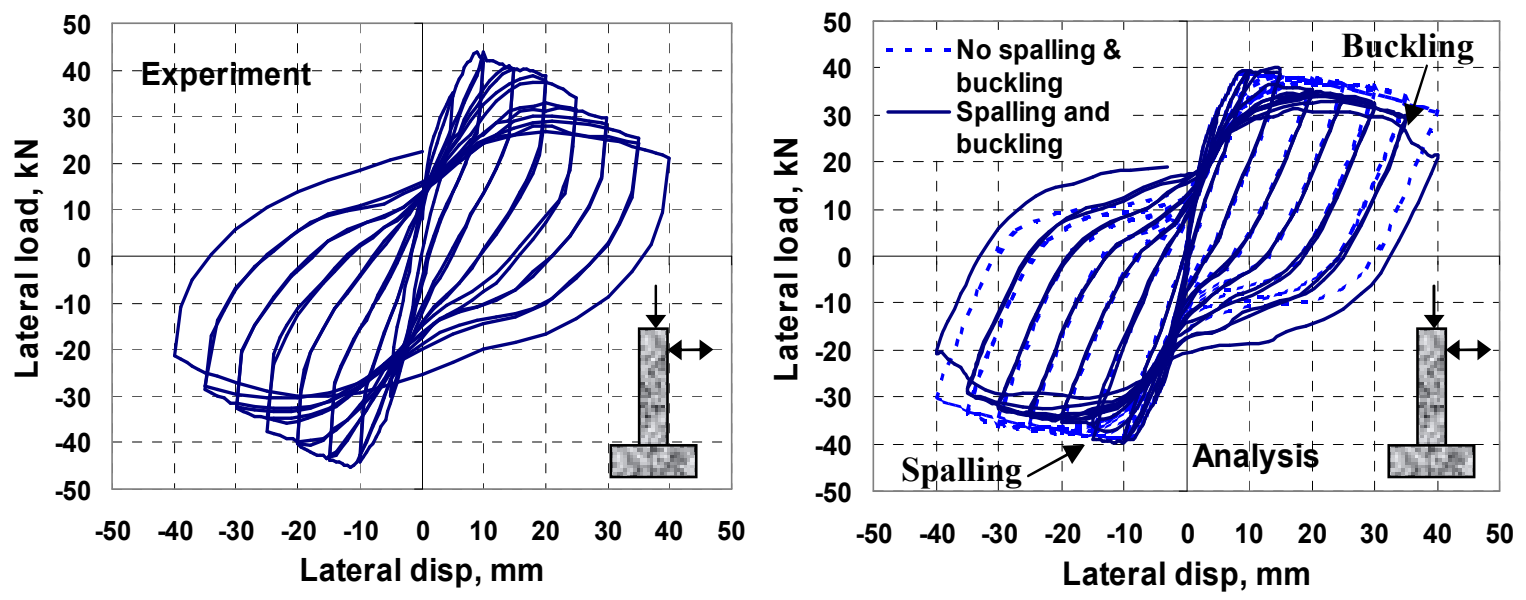
TABLE 1. Required Spring Stiffness for Different Buckling Modes

\begin{tabular}{|c|c|c|c|c|}
\hline \multirow{2}{*}{$\begin{array}{c}\text { Stable } \\
\text { buckling } \\
\text { mode, } \mathrm{n} \\
(1)\end{array}$} & \multicolumn{2}{|c|}{$\begin{array}{c}\text { Equivalent required stiffness } \mathrm{k}_{\mathrm{eq}} \\
\text { (Lateral ties eliminated in) }\end{array}$} & $\begin{array}{c}\text { Average } \\
\text { of (2) and } \\
(3)\end{array}$ \\
\cline { 2 - 5 } $\mathrm{k}_{\mathrm{eq}}$ \\
\hline 1 & 0.7500 & 0.7500 & 0.7500 & 0.7500 \\
\hline 2 & 0.1649 & 0.1649 & 0.1649 & 0.1649 \\
\hline 3 & 0.0976 & 0.0976 & 0.0371 & 0.0976 \\
\hline 4 & 0.0758 & 0.0137 & 0.0137 & 0.0448 \\
\hline 5 & 0.0084 & 0.0084 & 0.0084 & 0.0084 \\
\hline 6 & 0.0063 & 0.0063 & 0.0032 & 0.0063 \\
\hline 7 & 0.0052 & 0.0022 & 0.0022 & 0.0037 \\
\hline 8 & 0.0046 & 0.0016 & 0.0016 & 0.0031 \\
\hline 9 & 0.0013 & 0.0013 & 0.0008 & 0.0013 \\
\hline 10 & 0.0011 & 0.0006 & 0.0006 & 0.0009 \\
\hline
\end{tabular}


TABLE 2. Specimen Details

\begin{tabular}{|c|c|c|}
\hline $\begin{array}{c}\text { Properties } \\
(1)\end{array}$ & $\begin{array}{c}\text { Prism } \\
(2)\end{array}$ & $\begin{array}{c}\text { Column } \\
(3)\end{array}$ \\
\hline Cross-section & $20 \times 20 \mathrm{~cm}$ & $2.4 \times 2.4 \mathrm{~m}$ \\
\hline Main bar & 6 -D13 & $72-\mathrm{D} 35$ \\
\hline Stirrups & D6@10 cm & D19@30 cm \\
\hline $\begin{array}{c}\text { Number of } \\
\text { bars }\left(\mathrm{n}_{\mathrm{b}}, \mathrm{n}_{\mathrm{l}}\right)\end{array}$ & 6,2 & 19,2 \\
\hline $\begin{array}{c}\text { Length of tie } \\
\text { leg }\left(\mathrm{l}_{\mathrm{e}}\right)\end{array}$ & $16 \mathrm{~cm}$ & $220 \mathrm{~cm}$ \\
\hline $\begin{array}{c}\text { Young } \\
\text { modulus }\left(\mathrm{E}_{\mathrm{t}}\right)\end{array}$ & $200 \mathrm{Gpa}$ & $200 \mathrm{Gpa}$ \\
\hline $\begin{array}{c}\text { Yield } \\
\text { strength }\left(\mathrm{f}_{\mathrm{y}}\right)\end{array}$ & $355 \mathrm{Mpa}$ & $424 \mathrm{Mpa}$ \\
\hline $\begin{array}{c}\text { Equivalent } \\
\text { stiffness }\left(\mathrm{k}_{\mathrm{eq}}\right)\end{array}$ & 1.126 & 0.1015 \\
\hline Mode, $\mathrm{n}$ & 1 & 3 \\
\hline
\end{tabular}


TABLE 3. Calculation of Buckling Mode and Comparison with Experimental Observation

\begin{tabular}{|c|c|c|c|c|c|c|c|c|c|c|c|c|c|}
\hline 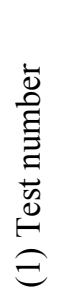 & 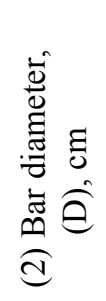 & 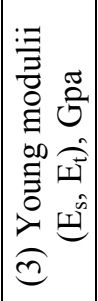 & 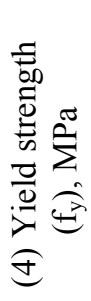 & 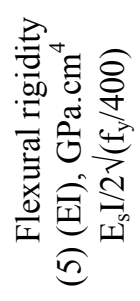 & 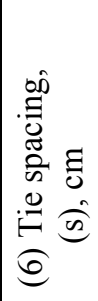 & 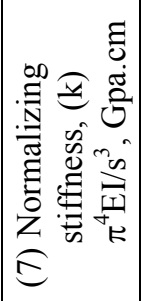 & 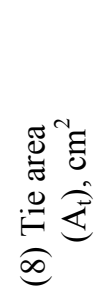 & 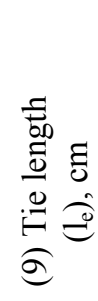 & 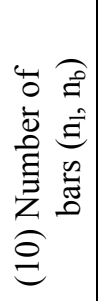 & 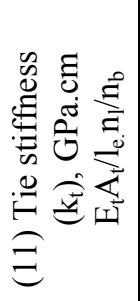 & 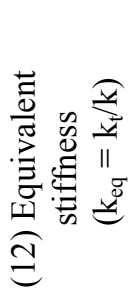 & 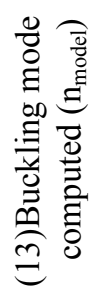 & 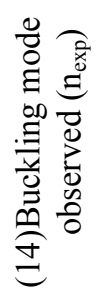 \\
\hline 1 & 1.563 & 200 & 438 & 30.66 & 6.3 & 11.94 & 0.691 & 24.3 & 2,3 & 3.785 & 0.317 & 2 & 1 \\
\hline 2 & 2.188 & 200 & 478 & 122.98 & 7.6 & 27.29 & 0.691 & 27.98 & 2,3 & 3.293 & 0.121 & 3 & 3 \\
\hline 3 & 2.188 & 200 & 478 & 122.98 & 7.6 & 27.29 & 1.227 & 29.89 & 2,3 & 5.474 & 0.201 & 2 & 3 \\
\hline 4 & 1.563 & 200 & 445 & 30.86 & 20 & 0.38 & 0.307 & 16.25 & 2,4 & 1.888 & 5.024 & 1 & 1 \\
\hline 5 & 1.563 & 200 & 445 & 30.86 & 20 & 0.38 & 0.307 & 16.25 & 4,6 & 2.517 & 6.699 & 1 & 1 \\
\hline 6 & 1.59 & 200 & 342 & 29.01 & 9.3 & 3.51 & 0.713 & 13 & 2,4 & 5.487 & 1.562 & 1 & 1 \\
\hline 7 & 1.27 & 200 & 343 & 11.83 & 9.3 & 1.43 & 0.713 & 13 & 2,4 & 5.487 & 3.832 & 1 & 1 \\
\hline 8 & 0.953 & 200 & 379 & 3.94 & 9.3 & 0.48 & 0.713 & 13 & 2,4 & 5.487 & 11.49 & 1 & 1 \\
\hline 9 & 1.59 & 200 & 342 & 29.01 & 7 & 8.24 & 0.317 & 13 & 2,4 & 2.436 & 0.296 & 2 & 3 \\
\hline 10 & 1.27 & 200 & 343 & 11.83 & 7 & 3.36 & 0.317 & 13 & 2,4 & 2.436 & 0.725 & 2 & 2 \\
\hline 11 & 0.953 & 200 & 379 & 3.94 & 7 & 1.12 & 0.317 & 13 & 2,4 & 2.436 & 2.176 & 1 & 1 \\
\hline 12 & 1.59 & 200 & 342 & 29.01 & 4.7 & 27.22 & 0.317 & 13 & 2,4 & 2.436 & 0.089 & 4 & 4 \\
\hline 13 & 1.27 & 200 & 343 & 11.83 & 4.7 & 11.09 & 0.317 & 13 & 2,4 & 2.436 & 0.219 & 2 & 3 \\
\hline 14 & 0.953 & 200 & 379 & 3.94 & 4.7 & 3.70 & 0.317 & 13 & 2,4 & 2.436 & 0.659 & 2 & 3 \\
\hline 15 & 1.59 & 200 & 342 & 29.01 & 3.5 & 65.91 & 0.317 & 13 & 2,4 & 2.436 & 0.037 & 5 & 5 \\
\hline 16 & 1.27 & 200 & 343 & 11.83 & 3.5 & 26.87 & 0.317 & 13 & 2,4 & 2.436 & 0.091 & 4 & 4 \\
\hline 17 & 0.953 & 200 & 379 & 3.94 & 3.5 & 8.95 & 0.317 & 13 & 2,4 & 2.436 & 0.272 & 2 & 2 \\
\hline 18 & 1.59 & 200 & 342 & 29.01 & 4.7 & 27.22 & 0.126 & 13 & 2,4 & 0.967 & 0.035 & 5 & 5 \\
\hline 19 & 1.27 & 200 & 343 & 11.83 & 4.7 & 11.09 & 0.126 & 13 & 2,4 & 0.967 & 0.087 & 4 & 4 \\
\hline 20 & 0.953 & 200 & 379 & 3.94 & 4.7 & 3.70 & 0.126 & 13 & 2,4 & 0.967 & 0.261 & 2 & 3 \\
\hline 21 & 1.59 & 200 & 342 & 29.01 & 3.5 & 65.91 & 0.126 & 13 & 2,4 & 0.967 & 0.014 & 5 & 6 \\
\hline 22 & 1.27 & 200 & 343 & 11.83 & 3.5 & 26.87 & 0.126 & 13 & 2,4 & 0.967 & 0.036 & 5 & 5 \\
\hline 23 & 0.953 & 200 & 379 & 3.94 & 3.5 & 8.95 & 0.126 & 13 & 2,4 & 0.967 & 0.108 & 3 & 3 \\
\hline 24 & 1.59 & 200 & 342 & 29.01 & 2.3 & 232.25 & 0.126 & 13 & 2,4 & 0.967 & 0.004 & 7 & 7 \\
\hline
\end{tabular}




\begin{tabular}{|c|c|c|c|c|c|c|c|c|c|c|c|c|c|}
\hline 25 & 1.27 & 200 & 343 & 11.83 & 2.3 & 94.67 & 0.126 & 13 & 2,4 & 0.967 & 0.010 & 5 & 5 \\
\hline 26 & 0.953 & 200 & 379 & 3.94 & 2.3 & 31.55 & 0.126 & 13 & 2,4 & 0.967 & 0.030 & 5 & 3 \\
\hline 27 & 1.59 & 200 & 739 & 42.64 & 14 & 1.51 & 0.713 & 13 & 2,4 & 5.487 & 3.624 & 1 & 1 \\
\hline 28 & 1.27 & 200 & 978 & 19.97 & 14 & 0.71 & 0.713 & 13 & 2,4 & 5.487 & 7.740 & 1 & 1 \\
\hline 29 & 1.27 & 200 & 978 & 19.97 & 7 & 5.67 & 0.317 & 13 & 2,4 & 2.436 & 0.429 & 2 & 2 \\
\hline 30 & 1.59 & 200 & 343 & 29.05 & 7 & 8.25 & 0.317 & 13 & 2,4 & 2.436 & 0.295 & 2 & 2 \\
\hline 31 & 1.27 & 200 & 356 & 12.05 & 7 & 3.42 & 0.317 & 13 & 2,4 & 2.436 & 0.712 & 2 & 2 \\
\hline 32 & 1.27 & 200 & 978 & 19.97 & 4.7 & 18.73 & 0.317 & 13 & 2,4 & 2.436 & 0.130 & 3 & 3 \\
\hline 33 & 1.27 & 200 & 356 & 12.05 & 4.7 & 11.30 & 0.317 & 13 & 2,4 & 2.436 & 0.215 & 2 & 3 \\
\hline 34 & 1.59 & 200 & 739 & 42.64 & 3.5 & 96.88 & 0.317 & 13 & 2,4 & 2.436 & 0.025 & 5 & 4 \\
\hline 35 & 1.27 & 200 & 978 & 19.97 & 3.5 & 45.37 & 0.317 & 13 & 2,4 & 2.436 & 0.054 & 4 & 3 \\
\hline 36 & 1.59 & 200 & 343 & 29.05 & 3.5 & 66.00 & 0.317 & 13 & 2,4 & 2.436 & 0.037 & 5 & 4 \\
\hline 37 & 1.27 & 200 & 356 & 12.05 & 3.5 & 27.37 & 0.317 & 13 & 2,4 & 2.436 & 0.089 & 4 & 3 \\
\hline 38 & 0.953 & 200 & 379 & 3.94 & 3.5 & 8.95 & 0.317 & 13 & 2,4 & 2.436 & 0.272 & 2 & 2 \\
\hline 39 & 1.27 & 200 & 978 & 19.97 & 4.7 & 18.73 & 0.126 & 13 & 2,4 & 0.967 & 0.051 & 4 & 6 \\
\hline 40 & 1.27 & 200 & 978 & 19.97 & 3.5 & 45.37 & 0.126 & 13 & 2,4 & 0.967 & 0.021 & 5 & 7 \\
\hline 41 & 1.27 & 200 & 978 & 19.97 & 2.3 & 159.86 & 0.126 & 13 & 2,4 & 0.967 & 0.006 & 7 & 7 \\
\hline 42 & 3.49 & 200 & 424 & 749.77 & 30 & 2.70 & 2.865 & 219.6 & 2,19 & 0.275 & 0.101 & 3 & 3 \\
\hline 43 & 3.49 & 200 & 424 & 749.77 & 15 & 21.64 & 2.865 & 219.6 & 4,19 & 0.549 & 0.025 & 5 & 4 \\
\hline 44 & 1.27 & 200 & 363 & 12.16 & 10 & 1.18 & 0.713 & 20 & 2,3 & 4.755 & 4.013 & 1 & 1 \\
\hline 45 & 1.27 & 200 & 355 & 12.03 & 10 & 1.17 & 0.317 & 16 & 2,6 & 1.320 & 1.126 & 1 & 1 \\
\hline
\end{tabular}

Source: Scribner (1986) $)^{1-3}$; Bresler and Gilbert $(1961)^{4-5}$; Kato et al. $(1995)^{6-41}$; PWRI (1999) $)^{42-43}$; Authors ${ }^{4-45}$ 
TABLE 4. Experimental Parameters

\begin{tabular}{|l|l|}
\hline \multicolumn{1}{|c|}{$\begin{array}{c}\text { Parameters } \\
(1)\end{array}$} & \multicolumn{1}{c|}{$\begin{array}{c}\text { Values } \\
(2)\end{array}$} \\
\hline Cross section, mm & $250 \times 250$ \\
\hline Main reinforcing bars & 6 - D13 \\
\hline Lateral ties, mm & D10@100 \\
\hline Cover thickness, mm & 30 \\
\hline Axial Load, kN & 250 \\
\hline Shear span, mm & 1200 \\
\hline Concrete strength $\mathrm{f}_{\mathrm{c}}{ }^{\prime}, \mathrm{MPa}$ & 28.6 \\
\hline Young's modulus $\mathrm{E}_{\mathrm{s}}, \mathrm{GPa}$ & 202 \\
\hline Yield strength $\mathrm{f}_{\mathrm{y}}, \mathrm{MPa}$ & 360 \\
\hline
\end{tabular}

